\title{
MULTICHANNEL COHERENT RADAR DEPTH SOUNDER FOR NASA OPERATION ICE BRIDGE
}

\author{
Lei Shi, Christopher T. Allen, John R. Ledford, Fernando Rodriguez-Morales, William A. Blake, \\ Ben G. Panzer, Stephen C. Prokopiack, Carlton J. Leuschen, and Sivaprasad Gogineni
}

\author{
Center for Remote Sensing of Ice Sheets \\ The University of Kansas
}

\begin{abstract}
The Multichannel Coherent Radar Depth Sounder (MCoRDS) system was developed by the Center for Remote Sensing of Ice Sheets (CReSIS) to map the thickness of ice sheets. This radar system was used in Antarctica as one of the primary sensors for NASA's Operation Ice Bridge (OIB) during the fall of 2009. Compared to its predecessors, MCoRDS features several new capabilities which enabled it to successfully capture ice thickness measurements over multiple glaciers on an aerial platform. This paper will focus on the capabilities of MCoRDS and also provide a sample of the processed radar results.
\end{abstract}

\section{INTRODUCTION}

NASA's ICESat (Ice, Cloud, and land Elevation Satellite) is an Earth Observation System that has been operating from 2003 to 2009 measuring ice sheet surface elevation and other parameters. The next generation of this satellite, ICESat-2, is currently scheduled to deploy in late 2015 [1]. This results in a time gap of 5 years where critical ice sheet data about rapidly changing ice streams would be lost [2].

Operation ICE Bridge (OIB) was launched to fill this data gap. For this project, NASA's DC-8 airborne laboratory aircraft is equipped with a variety of instruments to investigate the Arctic and Antarctic [2]. One of the primary instruments used during the first OIB field season in Antarctica was the Multichannel Coherent Radar Depth Sounder (MCoRDS) system. MCoRDS is an ice penetrating radar system used to measure ice thickness. It is the latest system developed by CReSIS at the University of Kansas for this mission and for other aerial platforms as well.

\section{SYSTEM DESCRIPTION}

\subsection{Basic Parameters}

The MCoRDS system for OIB is a nadir-looking, fivechannel, monostatic radar system. The table 1 lists the basic radar parameters used for the majority of the flights.
Table 1: Basic MCoRDS radar parameters

\begin{tabular}{|c|c|c|}
\hline Parameter & Value & Units \\
\hline PRF & 9000 & $\mathrm{~Hz}$ \\
\hline Tx Signal Type & Linear up chirp & $\mu \mathrm{s}$ \\
\hline $\begin{array}{c}\text { Signal Duration } \\
\text { (Low altitude) }\end{array}$ & 1 and 10 & $\mu \mathrm{s}$ \\
\hline $\begin{array}{c}\text { Signal Duration } \\
\text { (High altitude) }\end{array}$ & 30 & $\mathrm{MHz}$ \\
\hline Center Freq & 193.9 & $\mathrm{MHz}$ \\
\hline Bandwidth & 10 & $\mathrm{MSPS}$ \\
\hline Sampling Freq & 111 & $\mathrm{~W}$ \\
\hline Transmit Power & 500 & $\mathrm{~dB}$ \\
\hline Rx Noise Figure & 5 & Elements \\
\hline Antenna Element & $\lambda / 2$ bow-tie dipole & 5 \\
\hline Antenna Array & &
\end{tabular}

\subsection{Antenna array and fairing}

MCoRDS uses an antenna array consisting of five half-wave bow-tie dipoles for both transmitting and receiving signals. These antennas are mounted under the belly of the DC-8 aircraft using a custom fairing designed and fabricated by the department of Aerospace Engineering at the University of Kansas. The antennas inside the fairing are set up in a staggered collinear array pattern orthogonal to the direction of the flight path. A ground plane is also built into the fairing four inches above the antennas. Matching networks and W2DU baluns [3] were required for each antenna. Due to the extreme environment, the matching networks were placed inside the fuselage, directly above the antenna array.

Three addition monopole antennas are mounted inside the DC8 cabin. These antennas are connected to receive-only channels of the radar system and are used for monitoring electromagnetic interference (EMI) from other instruments. Matched-length cables were used for all five antennas in the fairing; however, different cables were used for the EMI antennas. The differences in these cable lengths were removed during data processing. 


\subsection{Radar system}

The radar itself is physically divided into four custom chassis; digital, transmit, power amplifier, and receiver chassis. These are mounted in a standard size, double bay rack inside the DC- 8 cabin. Figure 1 shows a high-level block diagram of the MCoRDS system.

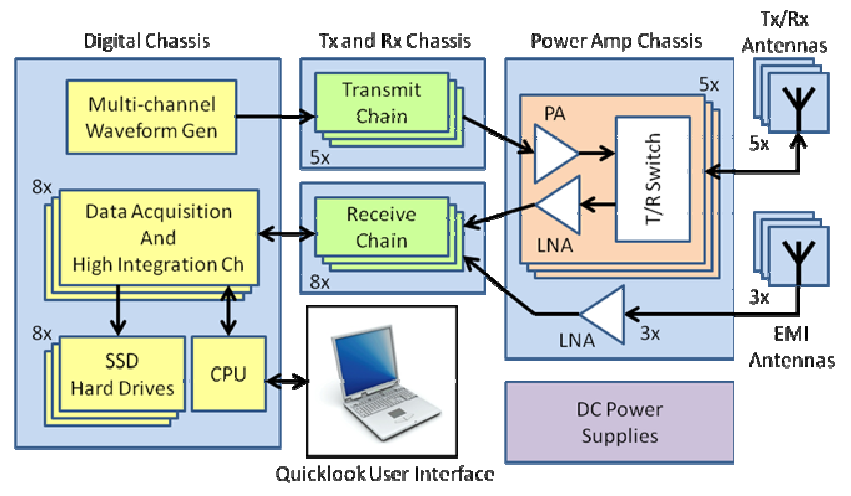

Figure 1: MCoRDS high-level block diagram

The digital chassis contains the controls for the entire radar system, including the three other chassis. Control signals from the digital chassis are transmitted to the other chassis via fiber optic cables for reduced EMI. The digital chassis also contains the hardware for signal generation, data acquisition, storage, and it provides the connection ports for the user interface laptop. The heart of this system is the eight channel waveform generator that creates the desired radar waveforms using direct digital synthesizers (DDS). These waveforms are transmitted to and from the digital chassis via coaxial cables with BNC connectors.

The transmit chassis filters and amplifies the waveform generated in the digital chassis. An off-the-shelf band-pass filter with a center frequency of $196 \mathrm{MHz}$ and a $40 \mathrm{MHz}$ bandwidth from KR Electronics was chosen. This filter eliminates undesired spectral components in the DDS output signal.

The power amplifier chassis contains two primary components: power amplifiers and T/R switches. A total of five $100 \mathrm{~W}$ linear power amplifiers are found here; one for each radar channel. This is the final gain stage before the waveform is transmitted. The $T / R$ switches toggle the antenna array from transmit to receive mode. Once in receive mode, the return echoes (captured using the same antenna array) is amplified by low noise amplifiers (LNAs) and fed into the receiver chassis.

The receiver chassis is similar to the transmit chassis with the addition of two programmable attenuators along the RF chain. At the end of each RF chain is an antialiasing filter made by Lorch Microwave. This filter has a center frequency of $195 \mathrm{MHz}$ and a 1-dB bandwidth of 30
MHz. After passing through this filter, the radar echo is sent to the digital chassis, where it is digitized and recorded onto eight solid state hard drives (SSDs).

\subsection{Eight channel waveform generator}

As mentioned earlier, the eight-channel waveform generator [4] (ECWG) is the heart of the digital chassis, which, in turn, controls the remainder of the radar. This unit was designed at CReSIS for beam-forming applications. It utilizes eight individual DDSs controlled by a single field programmable gate array (FPGA) to simultaneously generate up to eight waveforms. Each waveform can be independently controlled in terms of amplitude, frequency and phase, allowing true-time-delay beam steering.

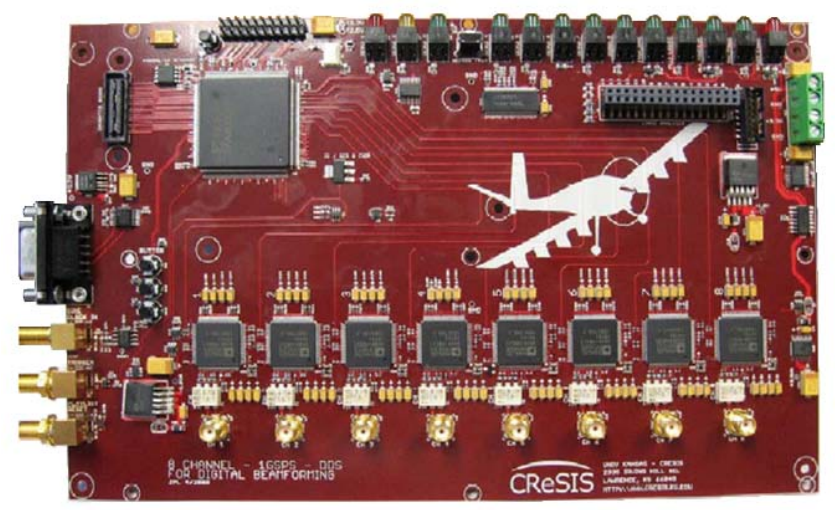

Figure 2: Eight-channel waveform generator board

A twenty percent Tukey waveform envelope is also applied to the DDS output chirp waveforms to improve time-sidelobe suppression. For the OIB missions, only five of the eight channels are needed. Since the radar is operated as nadir-looking, all five phases were calibrated to be identical. The programmable phase is also utilized to implement $0 / \pi$ inter-pulse modulation to cancel out coherent noise sources [5].

For the low altitude OIB flights, a playlist of multiple waveforms is loaded into the FPGA and generated by the DDSs in burst mode. On these flights, a single 1- $\mu$ s chirp waveform is first played to detect the surface, followed by sixteen $10-\mu$ s chirp waveforms to sound the bed. For high-altitude flights, a single $30-\mu$ s chirp waveform is used to capture echoes from both the surface and ice bed.

\subsection{Data acquisition}

During the receive window of a radar pulse repetition interval (PRI), the digital chassis also performs the task of digitizing and recording. Inside the digital chassis are eight data acquisition (DAQ) slices, one for each of the five radar channels and the three EMI channels. Analog-to-digital 
converters in each DAQ slice perform undersampling with a sample clock of $111 \mathrm{MHz}$. A buffer exists in each DAQ slice which is used to perform hardware presumming across the slow time axis before writing the final result to the SSDs. The number of hardware presums performed is in accordance with the play list loaded into the ECWG.

\section{RADAR USER INTERFACE}

A key development in the MCoRDS system over past CReSIS radars is in the new user interface (UI). Several iterations of the UI have been made since the OIB deployment; however, the main functions remain the same.

\subsection{Radar controls}

The parameters of the radar are controlled by a text file titled "config_radar.txt," which is loaded into the digital chassis from the user's laptop. This is a human-readable file that can be hand-modified to change all aspects of the radar performance, including, but not limited to DDS outputs, switch timing, variable attenuator settings, and even waveform additions or removals.

During the OIB missions, all files were manually modified and saved into the digital chassis to be loaded when desired. The latest version of the UI is able to generate the configuration file from scratch and automatically apply the changes to the radar.

\subsection{Quicklook}

One of the most useful features in the MCoRDS UI is called Quicklook. This software application performs real-time pulse compression that allowed the user to observe a small sample of the captured radar data written to the SSDs.

Each DAQ slice in the digital chassis has a buffer known as the "high integration channel," where a large amount of presums (100 or more) are performed by the

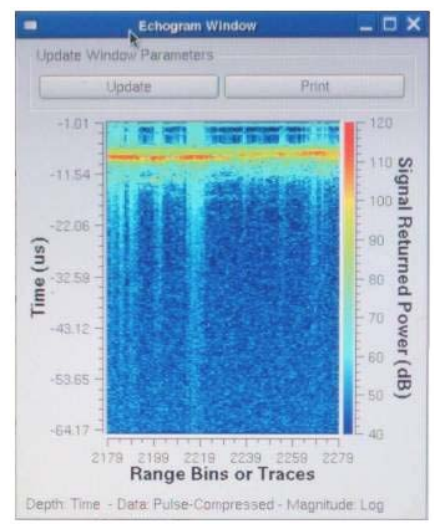

Figure 3: Quicklook scrolling echogram feature hardware (separate from the presumming performed before writing to SSDs). The data from this channel are read to the user's laptop once every one thousand loops of the full play list. These data are then stored on the user's laptop and can be displayed in real time via the Quicklook software as either a raw signal, pulse compressed signal, or scrolling echogram format. Pulse compression within Quicklook is performed using an ideal tapered chirp of the same bandwidth as the transmitted waveform.

\section{DATA PROCESSING}

The purpose of the MCoRDS radar is to make ice thickness measurements. To accomplish this, the radar data needs to be converted to echograms that show the surface and bottom of the ice. Initial data processing performed in the field uses the Quicklook data samples for a fast estimate of the echogram clarity. Upon returning from the field, a modified version of the CReSIS SAR Processor code (CSARP) is used to quickly generate preliminary data products. This processing code performs additional coherent integrations, pulse compression, and also generates an echogram for each of the waveforms on a per channel basis. Figures 4, 5 and 6 show example echograms from the $1-\mu$ s and $10-\mu$ s for all 8 channels.

Notice that the echogram from Figure 4 shows a strong surface echo while the echogram from Figure 5 shows a strong bed echo. Figure 6 is an EMI-channel echogram captured during the transmission of a $10-\mu$ s waveform. The straight line across the top of this echogram shows the transmission of the radar waveform. Directly under this line, it is observed that the surface return can also be identified.

Once the echograms are produced, they are loaded into a layer tracing program, also developed at CReSIS. Utilizing a combination of echograms, the surface and bottom ice layers are picked out, resulting in Figure 7 . The difference in time between the surface line and bed line is then directly converted to ice thickness. This data is available on the CReSIS website for the general public at

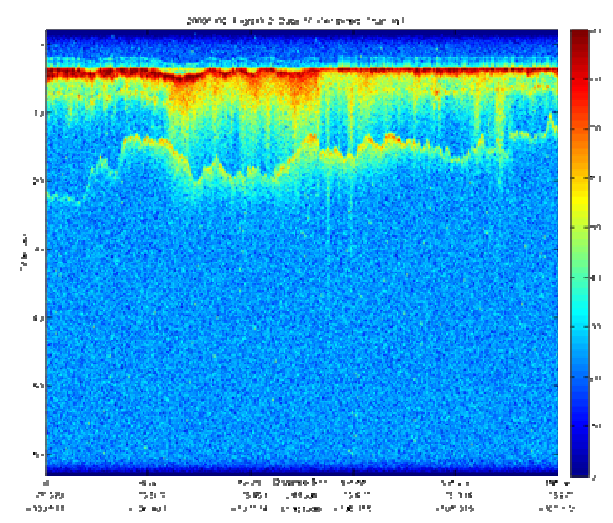

Figure 4: Example echogram from 1- $\mu$ s waveform 
www.cresis.ku.edu. Further processing is being performed on the entire data set, which will improve clarity and will fill in certain gaps that currently exist [6].

\section{CONCLUSION}

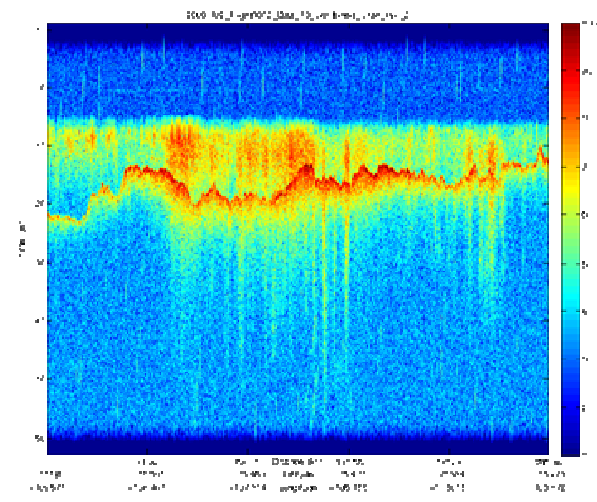

Figure 5: Example echogram from $10-\mu$ s waveform

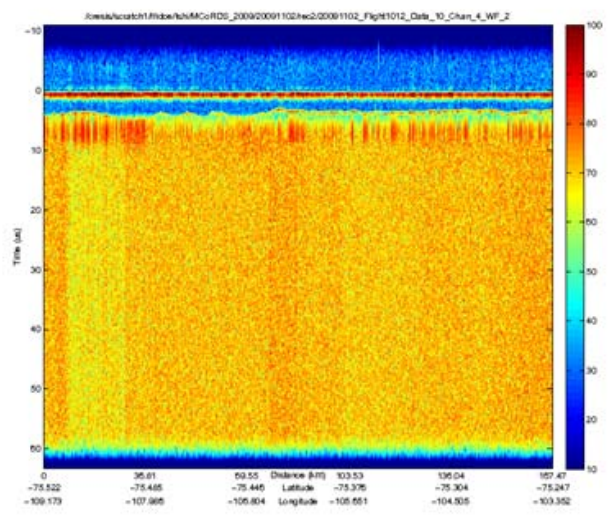

Figure 6: Example echogram from EMI channel

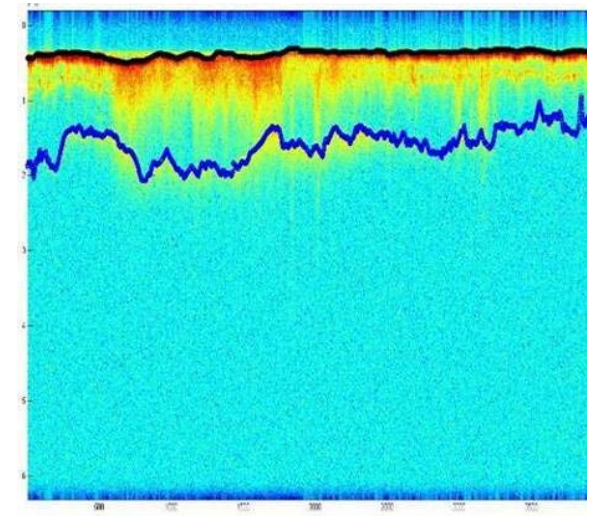

Figure 7: Layer tracing on an echogram
MCoRDS is a highly configurable system that is equipped with many features. Implementation of these key features is crucial in the successful operation of the radar system. Ice thickness measurements have been successfully captured across the majority of the OIB missions. More advanced processing techniques are being applied to areas of uncertainty and will ultimately be applied to the entire set of data. Since its deployment in the fall 2009 OIB Antarctica missions, modified version of MCoRDS have been deployed in airborne missions based in Antarctica as well as OIB Greenland missions.

\section{REFERENCES}

[1] National Aeronautics and Space Administration, "ICESat \& ICESat-2 Cryospheric Sciences Branch Code 614.1," National Aeronautics and Space Administration, May 2010. [Online]. Available: http://icesat.gsfc.nasa.gov. [Accessed: June 24, 2010].

[2] National Aeronautics and Space Administration, "Operation IceBridge Home Page," Earth Science Project Office, Jan. 2010. [Online]. Available: http://www.espo.nasa.gov/oib/ [Accessed: June 25, 2010].

[3] M. W. Maxwell, Reflections II: Transmission Lines \& Antennas, Worldradio Books, 2001.

[4] J. Ledford, "Development of an Eight Channel Waveform Generator for Beam-forming Applications." M.S. thesis, The University of Kansas, Lawrence, KS, US, 2009.

[5] C. T. Allen, S. N. Mozaffar, and T. L. Akins, "Suppressing Coherent Noise in Radar Applications With Long Dwell Times," IEEE Geoscience and Remote Sensing Letters, vol. 2, NO. 3, pp. 284-286. July 2005.

[6] W. Blake, L. Shi, J. Meisel, C. Allen, and P. Gogineni, "Airborne 3D Basal DEM and Ice Thckness Map of Pine Island Glacier,” IGARSS accepted, Hawaii, 2010. 